\title{
Uterine manipulation in operative laparoscopy by suction disposable uterine device
}

\author{
S. Tamburro E. Nouhz $\cdot$ F. Calonaci $\cdot$ A. Wattiez . \\ M. Canis • G. Mage
}

Received: 10 October 2010 / Accepted: 22 November 2010/Published online: 13 January 2011

(C) Springer-Verlag 2011

\begin{abstract}
The uterine mobilization is important to expose correctly the vesico-uterine plane, the adnexae, and the posterior cul-de-sac. Several studies compared different types of uterine manipulator and their maneuvrability, underlining the limitations or advantages related to the head and shaft when they are fixed together, limiting exposition or in the other, once there is dye injection capability. A new model of uterine manipulator by suction of endometrial cavity has been studied. Patients were not selected by uterine dimension or by gynecological disease; only the cases of endometrial cancer were excluded. In ten patients, we performed an hysteroscopic study associated to an endometrial specimen before installing the uterine device. No endometrial modification was found between normal endometrial specimen before surgery and after surgical treatment. The Vectec uterine manipulator has been demonstrated to be easy and safe to use.
\end{abstract}

\footnotetext{
S. Tamburro $(\bowtie) \cdot$ E. Nouhz $\cdot$ F. Calonaci

Department of Obstetrics \& Gynecology, CH Thiers,

Route du Fau,

63300 Thiers, France

e-mail: s.tamburro@cice.fr

\section{A. Wattiez}

CHU Hôpital Hautepierre, Chirurgie gynécologique,

1 Avenue Molière,

67098 Strasbourg Cedex 2, France

S. Tamburro $\cdot$ E. Nouhz $\cdot$ M. Canis $\cdot$ G. Mage

Department Obstetrics and Gynecology, CHU Clermont Ferrand,

CHU Estaing,

1 Place Lucie Aubrac,

63003 Clermont-Ferrand CEDEX 1, France
}

Keywords Laparoscopy · Uterine manipulator · Suction · New device

\section{Introduction}

Our aim and objective is to evaluate the efficacy and ergonomy of a new uterine manipulator and of the consequences of a suction system on endometrial tissue.

Laparoscopic surgery is currently accepted worldwide as an excellent diagnostic and treatment tool for many gynecologic problems. The uterine manipulator is essential, assisting the control of the uterus during a laparoscopic operation.

The uterine mobilization is important to expose correctly the vesico-uterine plane, the adnexae, and the posterior cul-desac. Otherwise, peritoneal lesions or defect of anterior and posterior space may not be evidenced and treated. In addiction pushing and lateralizing the uterus, more anatomical structures and spaces are visualized, such as paravesical and pararectal spaces. They could be accessible to dissection if necessary. Several studies compared different types of uterine manipulator and their maneuvrability, underlining the limitations or advantages related to the head and shaft when they are fixed together, limiting exposition or in the other once there is dye injection capability [2]. The uterine manipulator play the major role during operative laparoscopy approach showing uterine ligaments, parametrial spaces, uterine vessels, and to make colpotomy and hysterectomy; however, also during laparoscopy for adnexal pathology, it is revealed important to expose pelvic space $[1-3,5,6]$. 
More recently, in 2006, Banerjee, for the first time, suggested a suction cannula as uterine manipulator [4]. According to this idea, we developed a new model of uterine manipulator by suction of endometrial cavity, easy to install with the possibility to make a dye test. As part of a gynecologic procedure, dye is injected into the uterine cavity to observe its passage through the fimbria for evaluation of the fallopian tube patency in infertile patient [7-9].

\section{Materials \& methods}

The design of the study has been reported here. Ten patients, between 25 and 50 years old, were included in the study independently of uteri dimensions or previous surgery. Before the cannulation, an endometrial biopsy with a Cornier pipelle and a diagnostic hysteroscopy were performed in all patients to assess the endometrium. The Pipelle endometrial sampler obtains a small sample of endometrium for histological assessment without dilatation of the cervix, and as there is usually minimal discomfort associated with the procedure, it may be conducted in the outpatient setting. Tissue samples obtained were collected into $10 \%$ neutral buffered formalin, routinely processed in the pathology laboratory and embedded in paraffin. About 5-mm tissue sections were cut, mounted, dewaxed, and stained with haematoxylin and eosin. No uterine cervical forceps or cervical dilatation was necessary to install the uterine vacuum manipulator (Fig. 1).

Uteri dimension study by Vectec, hysterometer is made to adapt the manipulator to endometrial cavity with or without the use of a reducer device. The blunt upper extremity of the manipulator with holes is inserted in the uteri and connected to the vacuum by a tube with a luer lock link. Particular attention is necessary to push closely in contact the extremity of Vectec manipulator with the cervix to obtain the good vacuum and to solidarize it with uteri. The dye test was made, blocking the tap on manipulator and linking a syringe to inject methylene blue inside.

After surgical laparoscopic procedure, at the end of the surgery, the uterine manipulator has been removed, and a second endometrial study by Cornier device and diagnostic hysteroscopy were performed, as previously described above.

Adequacy of both preparations was assessed as satisfactory when sufficient cellular material was present to make a pathological diagnosis, or to exclude a pathological process with confidence.

After the use of a manipulator, we have focused the attention to compare the endometrial glands and stromal cells characteristics, cytoplasm, and basal lamina; in the vessels' endothelial structure and integrity, in luminal gland secretion, and inflammatory infiltration cells.

Specimens described as "barely adequate" contained scanty endometrial material that was adequate for assessment, but suboptimal for exclusion of pathology.

In contrast, a specimen was assessed as "inadequate" if there was insufficient cellular material in both the cytological slide and the cell block to exclude a pathological diagnosis. The stromal cells, the endometrial glands, vessels, and inflammatory cells infiltration were compared between the two specimens to study the modification caused by manipulator application.
Fig. 1 The Vectec manipulator: a hysterometer, b manipulator, c reducer device,

d vacuum tube

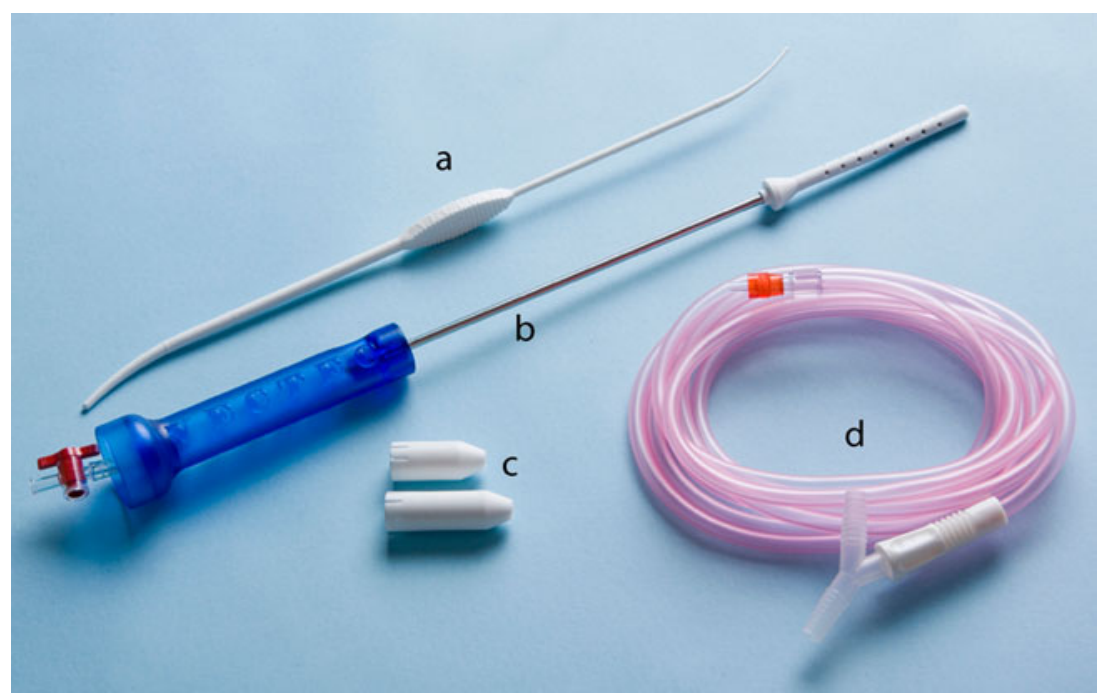




\section{Results}

No difficulties were found to insert the uterine manipulator, and the suction system allowed the surgeon to obtain a good exposition and mobility of uteri during surgery, independently of the uterine size. The Vectec manipulator has the advantage to be adaptable to each endometrial cavity; light and easy to mobilize. Using the manipulator, a blue dye test easily showed the bilateral tubal permeability, despite a fluid blue reflux which occurred around the cannula in three cases. In these cases, we checked that in a no right position of manipulator, which was not in contact with the uterine external orifice consequently, a non-optimal aspiration was obtained.

In the two groups of biopsies obtained before (Fig. 2, 3) and after manipulating, no differences were evidenced in specimens. Endometrial glands conserved their structure in multiple organization around the lumen, with the mucinous cell layers correctly represented. Glandular cells appeared normal, characterized with mucinous drops in cytoplasm and morphologically regular nuclei. The negative pressure induced by vacuum showed no modified basal cells layer and basal lamina of glands; however, in some lumen of glands, the gliar secretion induced by the suction action was visible. Among glands and stromal cells, mononuclear and other inflammatory cells were sometimes present to testify the consequences of suction induced by the manipulator, but no important distortions of tissue structure were found. (Fig. 4)

In all cases, no difference was found between two biopsies before and after uterine Vectec manipulator application. Tissue structures were well preserved, permitting an adequate analysis of tissue.

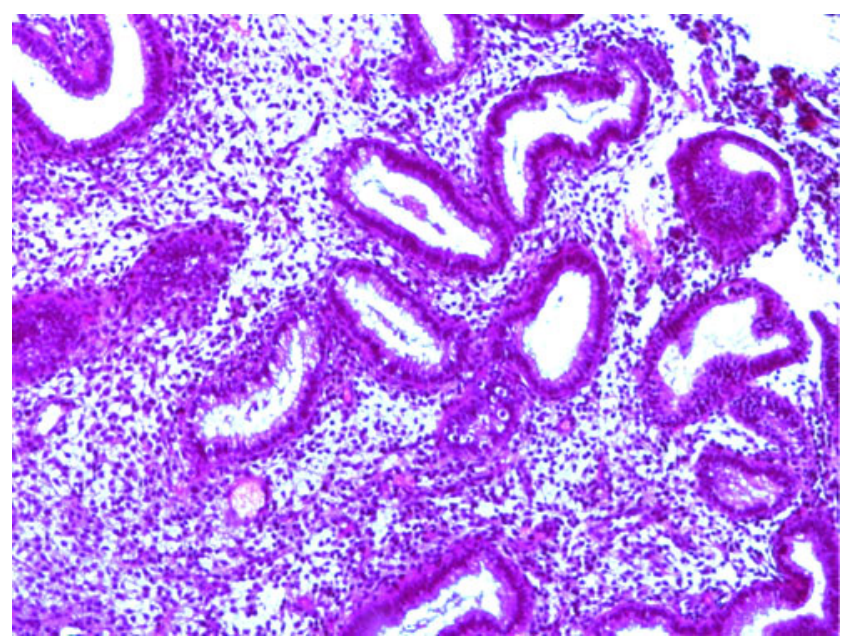

Fig. 2 Normal endometrial tissue before the use of the uterine manipulator

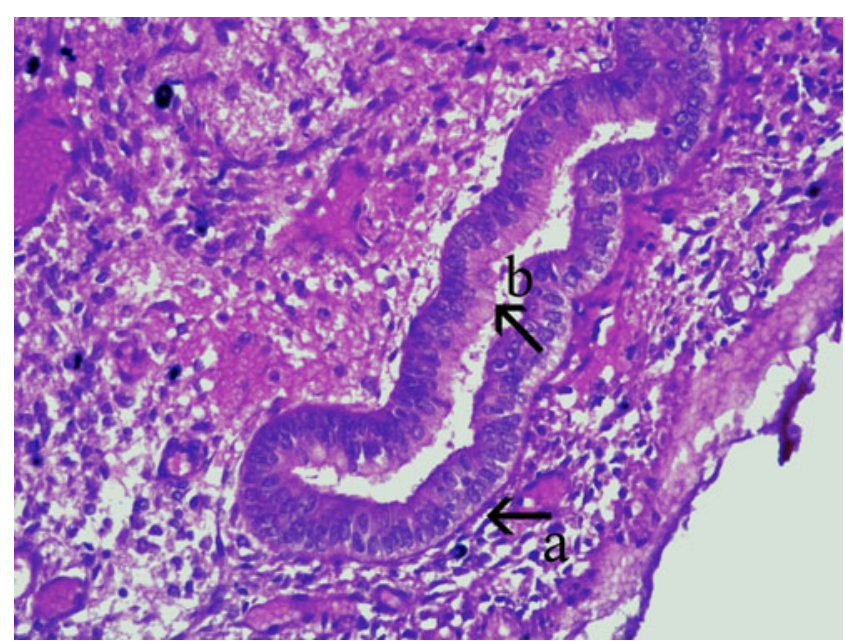

Fig. 3 Normal endometrial gland and stromal tissue. Basal layer (a) and mucinous fluid cells (b) are clearly evident (arrows)

\section{Conclusions}

The Vectec suction manipulator has demonstrated to be easy to install, cheap, and safe. The respect of hystological structure by suction is an important concept, because it could open other possibilities for others pathologies as application in oncology, where the integrity of tissue structures and cells is necessary to distinguish normal cells from atypical cells or neoplasia. In addition, the vacuum action in oncology could limit the neoplastic endometrial cells diffusion in peritoneal cavity during laparoscopic surgery. To evaluate this potential role, several studies are necessary which the results will be next published.

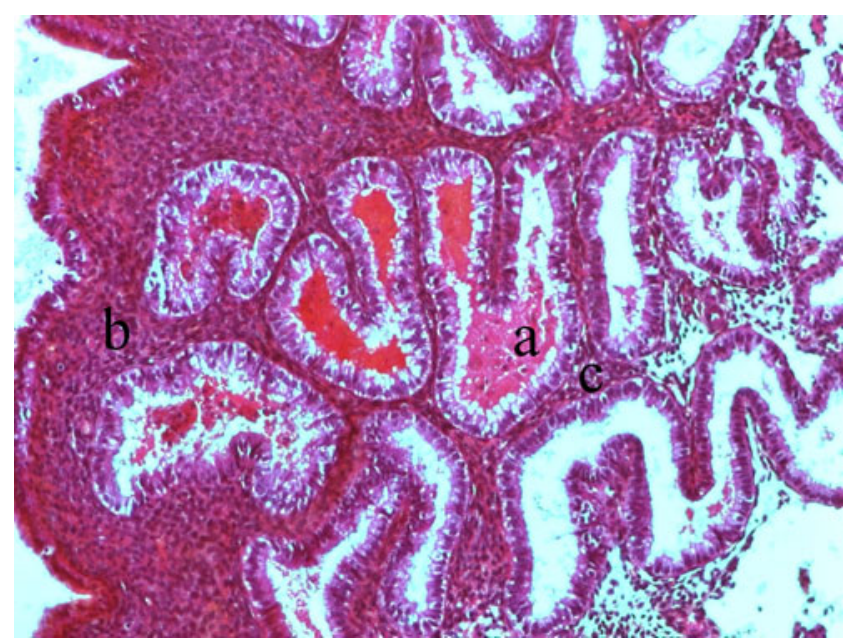

Fig. 4 Endometrium after manipulator use. Glands are intact with an important mucinous fluid in gland cavity (a); inflammatory cells are present among stromal cells (b). Basal layer is preserved and presented in normal structure (c) 
Declaration of interest The authors report no conflicts of interest. The authors alone are responsible for the content and writing of the paper.

\section{References}

1. Ramirez PT, Frumovitz M, Dos Reis R, Milam MR, Bevers MW, Levenback CF et al (2008) Modified uterine manipulator and vaginal rings for total laparoscopic radical hysterectomy. Int $\mathrm{J}$ Gynecol Cancer 18:571-575

2. Choksuchat C, Getpook C, Watthanagamthornkul S, Choobun T, Dhanaworavibul K, Tintara H (2008) Comparison of the TINTARA uterine manipulator with the Cohen cannula in gynecologic laparoscopy. J Med Assoc Thai 91:439-444
3. Agdi M, Tulandi T (2008) The benefits of intrauterine balloon: an intrauterine manipulator and balloon proved useful in myomectomy. A J Obstet Gynecol 199:581

4. Banerjee AK, Emembolu JO (2006) Plastic suction curette as uterine manipulator at combined laparoscopic sterilization: a prospective study of 531 cases. Eur J Contracept Reprod Health Care 11:310-313

5. Wu HH, Yeh GP, Hsieh TC (2005) Iatrogenic uterine rupture caused by overinflation of RUMI manipulator balloon. J Minim Invasive Gynecol 12:174-176

6. Bernstein P (1995) A new uterine manipulator for operative laparoscopic hysterectomy. J Am Assoc Gynecol Laparosc 2:331-333

7. Gregersen E (1994) The U-elevator. A new manipulator for gynecologic laparoscopy. Acta Obstet Gynecol Scand 73:508-510

8. Letterie GS (1991) Simple modification of an intrauterine manipulator. Int J Fertil 36:358-359

9. Scott JW (1983) A new uterine manipulator for use at laparoscopy. Am J Obstet Gynecol 147:458-459 